\title{
On central vision activity and peripheral sensitivity: A method with potentialities for research on a range of problems in visual perception
}

\author{
M. MASHOUR and T. HOLMBERG \\ University of Stockholm, Stockholm S-106 91, Sweden
}

\begin{abstract}
Peripheral sensitivity of the human eye, $P_{\mathrm{g}}$, and the processing capacity of the central retina, $V_{c}$, are assumed to be inversely related. On this basis, a method was designed, whereby $P_{s}$ as a function of $V_{c}$ can be determined and a family of $P_{s}$ maps for the whole surface of the periphery can be obtained. This can be done by means of an experimental system consisting of a computer-based visual field, a hemisphere, on which tracking patterns with varying degrees of difficulty are displayed centrally $\left(V_{c}\right)$ and multivariate signals (to be detected) are simultaneously presented on the periphery (as indexes of $P_{s}$ ). Since the apparatus is capable of producing and controlling a great number of variables, it can, with slight or no modifications, be used to study a wide range of problems in the field of visual perception and attention.
\end{abstract}

The method of research and instrumentation described here was designed to study the capacity of the visual system for processing input information. Theoretically, it is postulated that $\mathrm{C}$, defined as the maximum number of visual patterns processed per unit time, is limited and, relative to the total amount of information simultaneously reaching the receptors, very small. This implies that vision is essentially a sequential rather than a parallel process (Mashour, 1967, 1974). Another implication is that if the capacity $\mathrm{C}$ is allocated to a given task (normally, a central one), so that it becomes totally exhausted, then the peripheral receptors would be insensitive to sensory input.

Peripheral sensitivity, $P_{s}$, is assumed to be inversely related to the rate of information processing (or the degree of attention) via the central retina, $V_{c}$, (where $\mathrm{V}_{\mathrm{c}} \leqslant \mathrm{C}$ ). To our knowledge, however, this relationship, $P_{s}=f\left(V_{c}\right)$, has not been investigated, although peripheral sensitivity as such has been studied rather extensively. The relationship mentioned above represents the most natural and ordinary way of seeing, whereas the other way, seeing peripherally, occurs very seldom and requires training for concentration of attention.

\section{PRINCIPLES OF THE METHOD}

The central vision area (CVA) consists primarily of

This project was financed by the Swedish Council for Research in Humanities and Social Sciences. The authors wish to express their appreciation to Klas Barklöf, Bernard Devine, Göran Hagert, and Jim Nordin for valuable assistance. The authors' address is: Department of Psychology, University of Stockholm, Frescati Hagväg 14, S-106 91, Stockholm, Sweden. the fovea and, to some extent, the parafovea. In an experimental situation, CVA can be defined operationally by the size of the visual patterns presented centrally and sequentially. The rest of the retinal surface around CVA may, then, be regarded as the periphery and should, except when a peripheral signal ( $P$ signal) appears there, remain homogeneous.

$P_{s}$ is a variable aspect of the luminous stimulus ( $P$ signal), an index of sensitivity, that can be measured experimentally by having the observer, while he or she is performing the central task, report (by pressing a key) the presence or disappearance of a $P$ signal on the periphery.

$P_{s}$ can be determined for a sufficiently large number of points throughout the periphery across an experimentally acceptable range of $V_{c}$ values from $V_{c 1}$ to $\mathrm{V}_{\mathrm{cn}}$ (where $\mathrm{V}_{\mathrm{c} 1}<\mathrm{V}_{\mathrm{c} 2} \ldots<\mathrm{V}_{\mathrm{cn}}$ and $\mathrm{V}_{\mathrm{cn}} \approx \mathrm{C}$ ). In this way, a collection of $n$ maps of $P_{S}$ measures can be obtained and compared. Each map consists of a number of concentric cycles (parallels), each parallel representing a given visual angle, $\theta_{\mathbf{i}}$. Since the retinal points lying along a given parallel, $\theta_{\mathbf{i}}$, are equidistant from the center of the retina, they are predicted to be equisensitive as well. This means that the measure of sensitivity of a given parallel, $\mathbf{P}_{\mathrm{s}(\theta \mathrm{i})}$, can be meaningfully compared with the respective parallels on the other maps (see above). There are, therefore, as many sensitivity functions, $P_{s(\theta)}=f\left(V_{c}\right)$, as there are experimentally discriminable parallels.

The system described here was designed and constructed primarily for the purpose of determining the sensitivity maps mentioned above. Since the system has potentialities for producing, controlling, and registering a great number of variables, it can also be used to study 


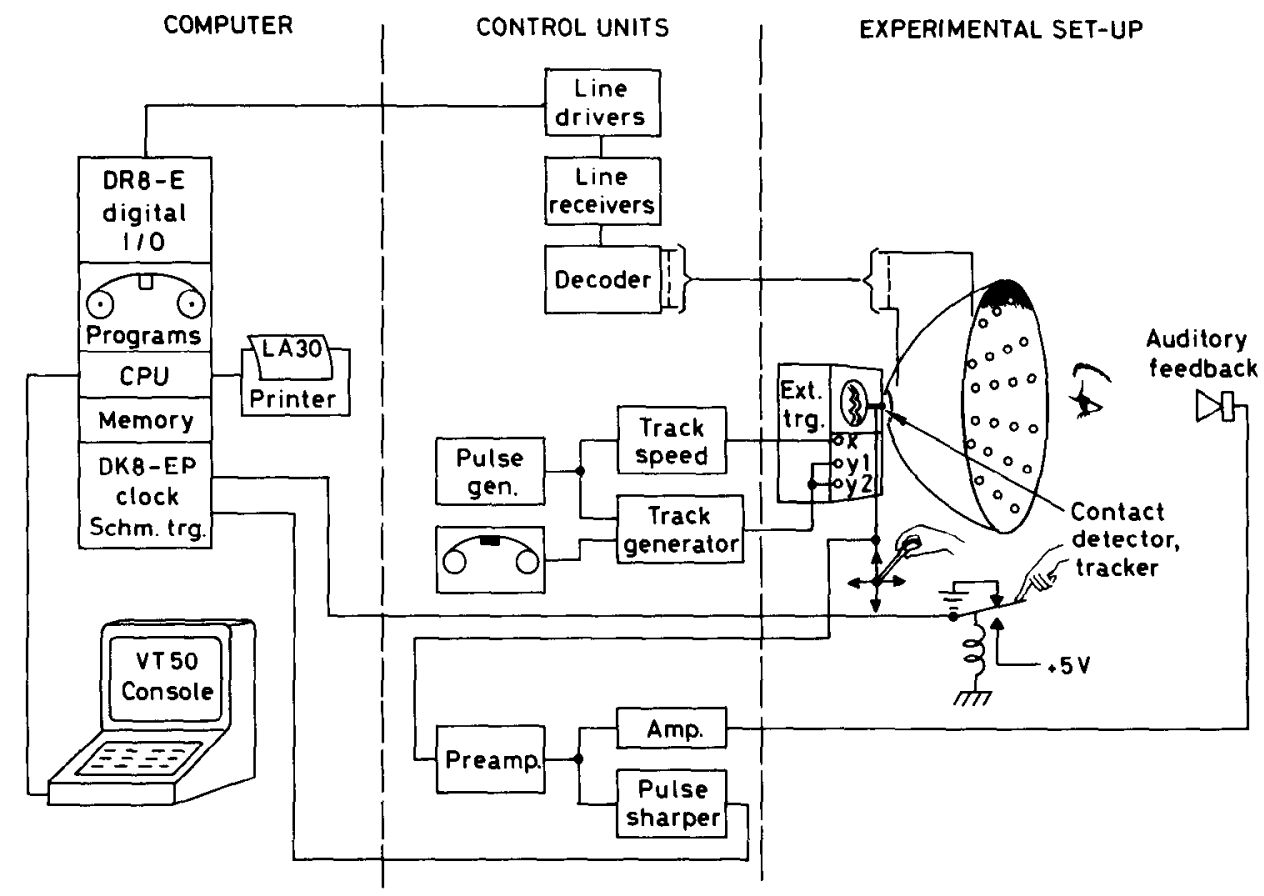

Figure 1. Schematic representation of the essential elements of operation of the experimental equipment, consisting of the computer and its complementary components, the control units, and the experimental setup. During the experiment, the subject's eye is positioned at the center of the hemisphere, the tracking target is steered with one hand, and the detection key is pressed with the other hand.

reaction time, attention, flicker, real and apparent movement, tracking, and other variables or tasks.

\section{EXPERIMENTAL SETUP AND APPARATUS}

The experimental system, illustrated in Figure 1, consists of the computer system, the experimental setup, and control units with electronic circuits adapted to the special functions required in the experiment.

\section{THE VISUAL FIELD}

The inner surface of a hemisphere (Figure 1) constitutes the visual field. The hemisphere is made of Plexiglas, $92 \mathrm{~cm}$ in diameter, colored gray, with $50 \%$ reflectance. The luminance of this surface can be adjusted by a series of incandescent lamps $(220 \mathrm{~V}, 25 \mathrm{~W})$ mounted behind an opaque edge along the border circumference of the hemisphere.

The $P$ signals consist of 192 luminous spots, $.3 \mathrm{~cm}$ in diameter, distributed at the intersection points of 16 imaginary parallels, $\theta$, and, orthogonal to these, 12 meridians, $\alpha$. The parallels are spaced $5 \mathrm{deg}$ apart (i.e., $\theta=5 \mathrm{deg}, 10 \mathrm{deg}, \ldots, 75 \mathrm{deg}, 80 \mathrm{deg}$ ), and the meridians are spaced $30 \mathrm{deg}$ apart $(\alpha=0 \mathrm{deg}, 30 \mathrm{deg}, \ldots$, $270 \mathrm{deg}, 300 \mathrm{deg}$ ). The intersections coincide with the endpoints of an equal number of perforations, all being directed toward the globe center. These apertures are covered with a special tape (reflectance 50\%) that distributes the incident light evenly. The light sources
(1.2.W bulbs) are mounted at the other end, opposite to the taped ends of the perforations. When required (e.g., in color vision research), the tape can easily be replaced by filters.

\section{VARIABLES}

By means of computer programs, it is possible to vary the following features of $P$ signals: $i=$ intensity level; $i_{\text {inc }}=$ stepwise increase of intensity from $i_{i}$ to $i_{j}\left(i_{i}<i_{j}\right)$ by equal or unequal steps, $\Delta i$, and vice versa $\left(i_{d e c}\right)$; $\mathrm{N}_{\Delta \mathrm{i}}=$ number of intensity steps; $\mathrm{t}_{\Delta \mathrm{i}}=$ duration of each step; $F F=$ flicker frequency from $i_{i}$ to $i_{j}$ and vice versa; $\mathrm{L} / \mathrm{D}=$ light-to-dark (time) ratio, a term used for a special case of FF, that is, when $i_{i}=0$ and $i_{j}>0 ; t_{b}=$ intersignal interval; and $\mathbf{N}_{\mathrm{s}}=$ number of signals presented simultaneously. By appropriately choosing the variable values in the combination $\Delta \mathrm{i} \times \mathrm{N}_{\Delta \mathrm{i}} \times \mathrm{t}_{\Delta \mathrm{i}}$, it is possible to create $P$ signals ranging perceptually from those smoothly increasing or decreasing in intensity, through signals with abrupt on-off behavior, to those constantly on.

Sequential presentations of signals, $t_{b}$, combined with the other temporal and intensity aspects mentioned above, provide appropriate conditions for a variety of experiments studying the effects of time-by-intensity variations, such as apparent movement.

In addition to the programmable variables mentioned previously, the following variables can be adjusted to the desired values: total number (density) of signals $\left(\mathrm{N}_{t}\right)$, 
general contrast level $\left(\mathrm{C}_{\mathrm{g}}\right)$ (by adjusting the incident light intensity of the globe surface), signal size, and signal and background colors (using filters and painting, respectively).

\section{CENTRAL VISION TASK}

The central vision area is demarcated by the side of an opening, a square, $4 \mathrm{~cm}$ (subtending an angle about $5 \mathrm{deg}$ at the center), in the pole of the hemisphere. Visual patterns of desired shapes, numbers, and so on, can be filmed and projected, or, using video technique (Reed's, 1979, report is particularly relevant), they can be shown on a TV screen at a suitable distance behind the opening. At this stage of the study, however, a sequence of curve patterns in a tracking task was preferred. This option was justified by the fact that the tracking variables were known and the patterns of a curve were relatively easy to control. The track patterns consist of a sequence of luminous curves moving on the oscilloscope screen.

The degree of difficulty (i.e., the rate of presenting patterns in sequence), $V_{c}$, can be controlled by one or a combination of the following variables: track width $(\mathrm{w})$, speed of movement (v), aspects of curvature ( $\mathrm{x}, \mathrm{y})$, contrast between the track and the screen $\left(C_{t o}\right)$, and target-track contrast $\left(\mathrm{C}_{\mathrm{tt}}\right)$. The observer's task in tracking is to follow the target along the curve and to adjust its position within the borders, using a two-dimensional lever. At the same time, he or she is required to report immediately upon the detection of a $P$ signal (s) by pressing a button.'

\section{THE TRACKING SYSTEM}

The tracking system consists of an oscilloscope (Gould Advance, Model OS 240), a detector of tracking errors, a steering mechanism for controlling the target, and circuits for the transmission of detector signals to the computer.

The cathode-ray tube (CRT) is dismounted from the oscilloscope set and mounted on a stand at a distance of $2.5 \mathrm{~cm}$ behind the opening. In this way, the CRT can revolve around its longitudinal axis and is adjustable to the desired position.

\section{Track Patterns and Track Generator}

A track can be produced in several ways, depending, among other things, on geometric characteristics. In the actual case, it is desirable to obtain a repeatable irregularly curved pattern sequence. The pattern's irregular feature is included to avoid learning effects in tracking.

In order to get a controlled pattern of movement, it is necessary that the sweep be started at exact time points relative to the curve pattern. This can be achieved if both the curve and the sweep are controlled by the same pulse generator (Figure 1). A sequence of the curve
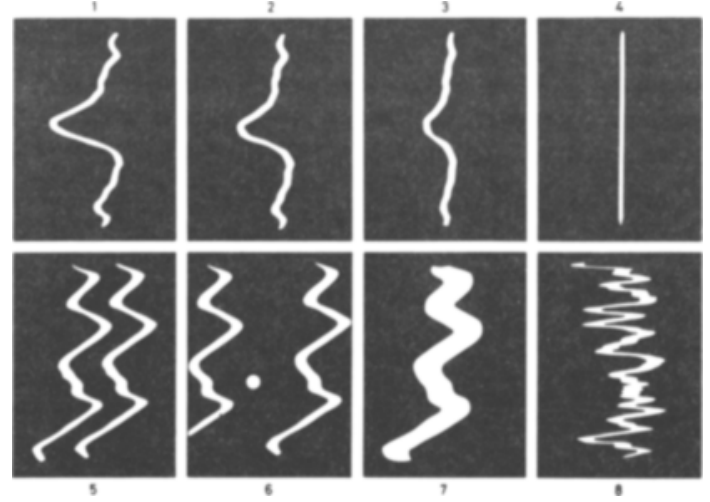

Figure 2. Photo frames showing a sample of sections of the track patterns: (a) two types of track, that is, one-line track $(1-4,7,8)$ and two-line track $(5,6)$; (b) variation in curvature (1-4); (c) width (compare $1-4$ with 7 and 5 with 6); (d) the tracking target (6); and (e) a compact longer sequence of patterns.

consists of 5,165 pulses and is repeated 75.5 times/sec. This means that if for every sequence of 5,165 pulses the sweep starts only once, the pattern on the CRT appears stationary (Figure 2).

In order to get the impression of movement, a track speed selector is used. This starts the sweep once every $5,170,5,175$, and 5,180 pulses (instead of 5,165 ). This results in three alternative movement rates (v), that is, $1.17,2.34$, and $3.51 \mathrm{cmS}^{-1}$, respectively, with the total time required for the whole sequence to run across the CRT screen amounting to about $14 \sec$ (at the lowest velocity).

Probable learning of the track curvature can, if necessary, be counteracted by lengthening the sequence and/or varying the curve amplitude continuously using modulating signals from a tape recorder. By means of computers, however, it is possible to program the desired figural patterns.

The track can be presented either as two delimiting luminous lines or as a single line (Figure 2). The latter alternative is brought about by combining the curve with a high-frequency triangular signal. In both cases, the track width can be varied at will between $.2 \mathrm{~cm}$ and $5 \mathrm{~cm}$.

\section{CONTACT DETECTOR}

The target consists of a luminous circle $(.18 \mathrm{~cm}$ in diameter) mounted at the nearer end of a metallic bar $(16 \times .2 \mathrm{~cm})$ in front of and perpendicular to the screen. The bar carries a phototransistor with a light-sensitive surface at the opposite end of the target at the immediate vicinity of the screen. When this sensitive surface (electronically) comes in contact with the track border (due to a tracking error) the contact is detected and signaled. In the present construction, however, the error is detected when about $50 \%$ of the sensitive surface lies 
outside the border. The error signals are then transmitted to three input gates in the Schmitt trigger logic.

\section{SIGNAL TRANSMISSION}

Transmission of signals from the control circuits to the computer occurs in parallel via a cable, an arrangement that might cause errors. This happens if differences in transmission time between the parallel lines exceeds the critical time value, 1 microsec. In order to prevent such disturbances, a cable of twisted-pair lines construction with terminal line drivers and line receivers was used (see Figure 1 and Figure 3B). Furthermore, a timing circuit (Figure $3 \mathrm{C}$ ) holds the fastest signals until the last one arrives, and then the circuit starts its operation.

\section{DECODER}

The choice and various functions of $\mathrm{P}$ signals (lamps) in the globe are controlled in the computer by a transmission capacity amounting altogether to 12 bits. Eight of these bits are involved in the selection processes of the 192 P signals. A decoder (Figure 3A) reads off the states of the eight outputs. Four of the 8 bits mentioned above are connected in parallel to 12 integrated circuits, IC1-IC12 (Model SN 74 154). Each of these circuits has four input gates, and these, in turn, are coded (using 4 bits) to 1 of the 16 outputs. Since these outputs are connected to lamp drivers (Figure 3D), one such circuit is capable of controlling the 16 lamps (P signals) lying along a given ray (meridian arc $\alpha$ ). Each of the circuits IC1-IC12 has a strobe input gate that prevents the out- put's being affected by the inputs before the strobe has received a signal for the execution of its function. Such signals originate from the corresponding outputs of IC13 (Figure 3A).

\section{Lamp Drivers}

As to the remaining 4 bits: Bits $0-2$ control the intensity of $P$ signals up to a maximum of 265 steps. Variation of intensity by these steps is too fine to be discriminated by the human eye. Finally, Bit 3 sets all lamp drivers to zero output, thereby extinguishing all signals.

\section{COMPUTER}

The computer is a PDP-8/e (Digital Equipment Corporation) that, besides processing and storing data and documentation, controls the special functions mentioned previously. It has been specially adapted to these functions with the circuits shown in the middle section of Figure 1. Moreover, the computer has been equipped with the following complementary components: buffered digital I/O DR8-E, real-time programmable clock DK8-EP, with Schmitt trigger logic and an alphanumeric CRT terminal, Model VT 50. The buffered digital $\mathrm{I} / \mathrm{O}$ has a 12-channel output flip-flop with parallel interface cable connections $1.5 \mathrm{~m}$ long. The clock is used for synchronizing external events, such as counting the number of errors in tracking and measuring time, as well as for controlling program interruption. During the experiment, the alphanumeric terminal is used as a console and the ordinary console, Model LA 30, as a typewriter.
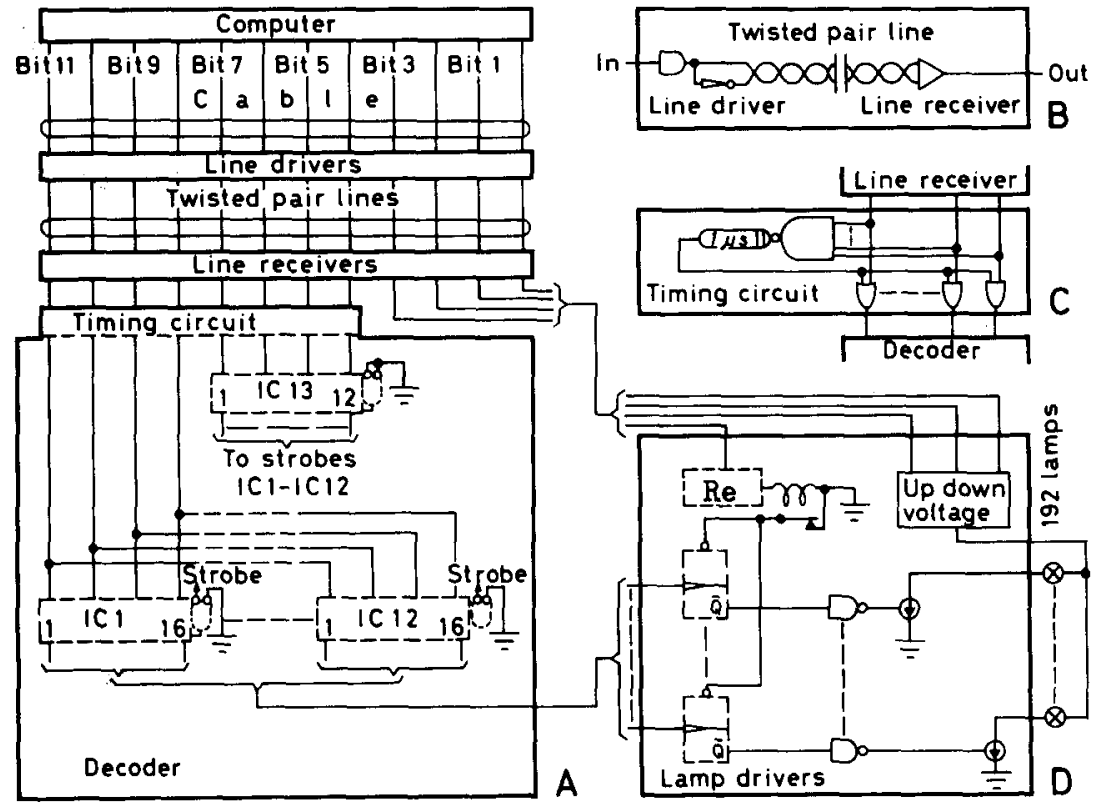

Figure 3. Diagram representing the mechanism for control and transmission of signals. "Re" in Section D denotes relay. 


\section{TO JOIN THE SYSTEM TO OTHER COMPUTERS}

It is possible to join the experimental system to modern and more powerful laboratory computers such as MINC-11 or MINC-23 in the MINC family, instead of PDP.8/e. This can be done by substituting the A/D converter for the Schmitt trigger logic (Figure 1) and replacing the DR8-E digital I/O and DK8-EP clock (Figure 1 ) by the corresponding optional I/O modules of the selected (micro) computer system.

There are also some computer systems, such as the Swedish $\mathrm{ABC}-80$ and $\mathrm{ABC}-800$, with built-in $\mathrm{I} / \mathrm{O}$ modules (see above). These systems can be connected to the experimental system directly.

\section{REFERENCES}

Mashoug, M. Information transmission in speed perception and locomotion. In F. Klix (Ed.), The organization of human information processing. Berlin: Akademie-Verlag, 1967.
Mashour, M. Human factors in signalling systems. New York: Wiley, 1974.

ReEd, A. V. Microcomputer display timing: Problems and solutions. Behavior Research Methods \& Instrumentation, 1979, 11, 572-576.

\section{NOTE}

1. The problem of localization of the peripheral stimulus (image) on the retina is a matter of averaging the data obtained for every signal. Since every signal should be presented repeatedly and sufficiently often, the target sometimes comes in contact with the left edge and sometimes with the right edge of the track resulting, for example, in higher or lower intensities. It is the average of these intensities that is attributed to a given peripheral signal. These averages are expected to be both statistically different for the signals along a given meridian and statistically equal for the signals along a given parallel. Otherwise, signals with proper spacing should be selected for the particular experiment. Problems such as control of eye movements by chin rest, bite bar, and so on, depend upon the experimental problem and design. This paper does not deal with specific designs.

(Received for publication January 5, 1982; revision accepted May 30, 1982.) 